\title{
AMCoR
}

Asahikawa Medical College Repository http://amcor. asahikawa-med. ac. jp/

NEUROSCIENCE RESEARCH (2003) 46(4):523-532.

Paraquat leads to dopaminergic neural vulnerability in organotypic midbrain culture

Shimizu, K; Matsubara, K; Ohtaki, K; Shiono, H 


\section{Paraquat leads to dopaminergic neural vulnerability in organotypic midbrain culture}

Keiko Shimizu a, Kazuo Matsubara b, ${ }^{*}$, Ko-ichi Ohtaki b, Hiroshi Shiono a

a Department of Legal Medicine, Asahikawa M edical College, Asahikawa 078-8510, Japan

b Department of Hospital Pharmacy \& Pharmacology, Asahikawa M edical College, Asahikawa 078-8510, Japan

* Corresponding author. Tel.: + 81-166-69-3480; Fax: + 81-166-65-1392

E-mail address: kmatsuba@asahikawa-med.ac.jp (K. Matsubara)

Text pages: 21; Table: 0; Figure: 7 
Abstract

Paraquat (PQ) is a herbicide to possibly induce Parkinson's disease (PD), since a strong correlation has been found between the incidence of the disease and the amount of paraquat used. In this study, we examined PQ toxicity in rat organotypic midbrain slice cultures. PQ dose dependently reduced the number of dopaminergic neurons in cultured slices. Since this damage was prevented by GBR-12909, the dopamine transporter could be an initial step of the PQ induced dopaminergic neurotoxicity. The sequential treatments with lower PQ and $\mathrm{MPP}^{+}$doses, where each dose alone was not lethal, markedly killed dopamine neurons, suggesting that the exposure of a lower dose of PQ could lead to the vulnerability of dopaminergic neurons. This cell death was prevented by the inhibitors of NMDA, nitric oxide synthase (NOS), cycloheximide and caspase cascade. Neurons expressing NOS were identified inside and around the regions where dopamine neurons were packed. The cell death induced by the sequential treatments with PQ and $\mathrm{MPP}^{+}$was also rescued by Ldeprenyl and dopamine D2/3 agonists. These results strongly support that the constant exposure to low levels of PQ would lead to the vulnerability of dopaminergic neurons in the nigrostriatal system by the excitotoxic pathway, and might potentiate neurodegeneration caused by the exposure of other substances and aging.

Keywords: Paraquat; Dopamine neurons; Organotypic midbrain slice; Neurotoxicity; Parkinson's disease 


\section{Introduction}

Parkinson's disease (PD) is one of the most common neurodegenerative diseases, and the prominent pathological feature of the PD brain is the selective deterioration of dopaminergic neurons in the substantia nigra pars compacta of the midbrain and a resultant decrease in dopamine levels in the striatum, the main target innervated by this neuronal population. After the discovery of 1-methyl-4-phenyl-1,2,3,6-tetrahydropyridine (MPTP), it is hypothesized that PD may be initiated or precipitated in genetically-predisposed individuals by environmental and/or endogenous toxins through a mechanism similar to that of MPTP (Betarbet et al., 2000; Brooks et al., 1999; Corrigan et al., 2000; Kotake et al., 1995; Maruyama et al., 1996; Matsubara et al., 1995; Sanchez-Ramos et al., 1998; Thiruchelvam et al., 2000a). Indeed, increasing evidence from epidemiological studies has implicated the possible involvement of environmental chemicals or endogenous substances (Kotake et al., 1995; Maruyama et al., 1996; Matsubara et al., 1995) in the selective dopaminergic cell loss in the substantia nigra of non-familial PD. Non-familial PD represents greater than $90 \%$ of all PD cases and its onset typically occurs after 50 years of age (Langston, 1998; Moghal et al., 1994; Olanow and Tatton, 1999; Tanner et al., 1999). Identified PD risk factors include herbicide and pesticide usage, farming and well-water consumption (Fleming et al., 1994; Seidler et al., 1996; Semchuk et al., 1993). A recent study of over 19,000 Caucasian male twins confirmed that genetic heritability is not the basis of sporadic PD with onset at greater than 50 years old (Tanner et al., 1999). In addition, there is increasing evidence that excitotoxic injury also plays a critical role in progressive dopaminergic degeneration (Beal, 1998; Dawson and Dawson, 1998; Dugan and Choi, 1999). The influx of $\mathrm{Ca}^{2+}$ induced by the activation of NMDA triggers the mobilization of $\mathrm{Ca}^{2+}$-dependent intracellular processes including the activation of neuronal nitric oxide synthase (NOS). Nitric oxide (NO) produced by NOS is suggested to play an important role in excitotoxicity, probably through the formation of peroxynitrite anion by reaction with superoxide radical (Canals et al., 2001; Gatto et al., 2000; LaVoie and Hastings, 1999; 
Obata and Yamanaka, 2001). The series of radicals act as mitochondrial toxins inducing selective dopaminergic cell death (Beal, 1998; Nakamura et al., 2001; Simonian and Coyle, 1996).

Paraquat (1,1'-dimethyl-4,4'-bipyridinium, PQ), is widely used as a non-selective herbicide, and bears structural similarity to 1-methyl-4-phenyl pyridinium $\left(\mathrm{MPP}^{+}\right)$. This herbicide adversely impacts on dopamine systems, and a strong geographical overlapping has been found between the incidence of PD and the amount of PQ used (Lanska, 1997; Liou et al., 1997; Rajput et al., 1987; Ritz and Yu, 2000). PQ induces dopaminergic neuronal damage in experimental animals (Corasaniti et al., 1992; Corasaniti et al., 1998). Interestingly, it has been demonstrated that the mixture of PQ and dithiocarbamate induces selective dopaminergic neurotoxicity (Thiruchelvam et al., 2000a; Thiruchelvam et al., 2000b). However, the impact of PQ on dopamine systems is equivocal, because its neurotoxic mechanism has not been well documented. PQ is translocated into the brain by the neutral aminoacid transporter, and then is taken up in brain tissues by dopmaine transporters (Shimizu et al., 2001; Shimizu et al., 2003). Furthermore, we have previously reported, by the microdialysis method, that PQ stimulated glutamate efflux to initiate excitotoxicity on dopaminergic terminals, resulting in continuous and long-lasting dopamine overflow (Shimizu et al., 2003). These findings indicate that constant exposure to low levels of PQ may lead to the vulnerability of dopaminergic neurons.

Thus, it is essential to investigate the toxic mechanism of PQ on the dopaminergic neurons in the substantia nigra. In this study, we studied PQ toxicity on the dopaminergic neurons in organotypic midbrain cultured slices. We also investigated the effect of sequential exposures of PQ and $\mathrm{MPP}^{+}$, where each dose alone was not lethal, on the dopamine neurons. The mechanism underlying the dopaminergic toxicity of PQ was evaluated using chemicals to inhibit NMDA receptor, NOS and caspase cascade. Furthermore, we evaluated whether the neural damage induced by the sequential exposures of PQ and $\mathrm{MPP}^{+}$was protected by L-deprenyl or dopamine D2/3 agonists. 


\subsection{Chemicals}

PQ dichloride was obtained from Tokyo Chemical Industry (Tokyo, Japan). MPP+ iodide, L-deprenyl and dizocilpine (MK-801) were purchased from Research Biochemicals International (Natick, MA, USA). GBR-12909 (1-(2-[bis(4-Fluorophenyl)methoxy]ethyl)4-(3-phenylpropyl)piperazine dihydrochloride) and cycloheximide, were obtained from Sigma (Saint Louis, MO, USA). Acetyl-L-leucyl-L-glutamyl-L-histidyl-L-aspart-1-al trifluoroacetate (caspase 9 inhibitor) and acetyl-L-aspartyl-L-methionyl-L-glutaminyl-Laspart-1-al (caspase 3 inhibitor) were purchased from the Peptide Institute (Tokyo, Japan). Cabergoline and talipexole were kindly gifted from Japan Pharmacia (Tokyo, Japan) and Japan Boehringer Ingelheim (Kawanishi, Japan), respectively. The other reagents were of analytical grade, and were from Wako (Osaka, Japan). GBR-2909 potently inhibits dopamine uptake and demonstrates a nearly 400-fold greater selectivity for the inhibition of dopamine over norepinephrine uptake in the synaptosomal preparation. MK-801 and dinitroquinoxaline-2,3-dione (DNQX) are highly potent and selective non-competitive NMDA and AMPA/kinate (non-NMDA), respectively, receptors. $\mathrm{NG}^{\mathrm{G}}$-nitro-L-arginine methyl ester (L-NAME) is an analog of arginine that inhibits NO production.

\subsection{Culture preparation}

Organotypic slice cultures were prepared according to the method of Stoppini et al. (1991) and Katsuki et al. (2001) with slight modifications. Briefly, a postnatal 2 - 3 days of Wistar rat was anesthetized by hypothermia, the brain was removed from the skull and separated into two hemispheres. Five coronal slices $(350 \mu \mathrm{m}$ thick) of the mesencephalon were prepared under sterile conditions with a tissue chopper, and transferred onto Millicell$\mathrm{CM}$ insert membranes in six-well plates. Culture medium, consisting of $50 \%$ minimum 
essential medium with HEPES buffer, 25\% Hank's balanced salt solution and 25\% heatinactivated horse serum supplemented with $6.5 \mathrm{mg} / \mathrm{ml}$ glucose and $2 \mathrm{mM}$ L-glutamine, was supplied at a volume of $1 \mathrm{ml}$ per well. The culture medium was exchanged with fresh medium every 2-day. Slices were maintained for 13 - 15 days in a $5 \% \mathrm{CO}_{2}$ humidified atmosphere at $34^{\circ} \mathrm{C}$ before drug treatment. The animal experiments were performed in accordance with the guidelines for care and use of laboratory animals by the Committee of Asahikawa Medical College.

\subsection{D rug treatments}

Cultured slices were exposed to various concentrations of PQ for 2 days. For the second experiment, cultured slices were also sequentially incubated with $10 \mu \mathrm{M}$ PQ for 2 days and then $1 \mu \mathrm{M} \mathrm{MPP}+$ for 1 day. In every experiment, slices from the contralateral hemisphere from the same animal were used as controls.

Several chemicals were tested to rescue the dopaminergic neurotoxicity induced by the $50 \mu \mathrm{M}$ PQ or the sequential exposures of $10 \mu \mathrm{M}$ PQ and $1 \mu \mathrm{M} \mathrm{MPP}^{+}$. These chemicals were pre-incubated for $2 \mathrm{~h}$ prior to the PQ exposure, and continuously exposed to the slices during the experiment days. Chemicals used were GBR-12909, L-NAME, MK-801, DNQX, caspase 9 inhibitor, caspase 3 inhibitor, cycloheximide, cabergoline, talipexole and L-deprenyl. These drugs were dissolved in the culture medium. Control slices were also administered these drugs without the toxin treatment.

\subsection{Tyrosine hydroxylase (TH) immunohistochemistry}

Dopaminergic neurons in the slice cultures were identified by tyrosine hydroxylase (TH) immunohistochemistry using the avidin-biotin peroxidase method. Cultured slices were fixed with $0.1 \mathrm{M}$ phosphate buffer containing $4 \%$ paraformaldehyde and $4 \%$ sucrose for $2 \mathrm{~h}$, and processed for $\mathrm{TH}$ immunohistochemistry according to the method of Maeda et 
al. (1998). After rinsing with phosphate-buffered saline (PBS), fixed slices were exposed to $0.02 \% \mathrm{H}_{2} \mathrm{O}_{2}$ in $100 \%$ methanol for $30 \mathrm{~min}$, then to $0.2 \%$ Triton $\mathrm{X}-100$ in PBS for 30 min. Slices were subsequently treated with $10 \%$ fetal calf serum for $30 \mathrm{~min}$, then incubated with rabbit anti-TH polyclonal antibody (1: 500 dilution) overnight at $4^{\circ} \mathrm{C}$. After incubation for $1 \mathrm{~h}$ at room temperature with biotinylated antirabbit $\mathrm{IgG}$, slices were rinsed with PBS, then treated for $1 \mathrm{~h}$ at room temperature with avidin-biotinylated horseradish peroxidase complex. After a further wash with $50 \mathrm{mM}$ Tris-buffered saline, peroxidase was visualized with $0.07 \%$ 3,3'-diaminobenzidine and $0.018 \% \mathrm{H}_{2} \mathrm{O}_{2}$. Specimens were dehydrated through a graded ethanol series and mounted on slide glasses with glycerol for observation under a bright-field microscope. Positively stained cells bearing developed dendrites that were at least greater than twice as long as the cell diameter were considered to be viable dopaminergic neurons. The maximal number of viable dopaminergic neurons in an area of $520 \times 670 \mu \mathrm{m}^{2}$ in individual slices was counted.

\subsection{NADPH diaphorase histochemistry}

Double staining of slice cultures with NADPH diaphorase and TH was performed in several slices. After rinsing with $0.1 \mathrm{M}$ phosphate buffer ( $\mathrm{pH} 7.4$ ), fixed cultures were incubated in $0.1 \mathrm{M}$ phosphate buffer containing $1 \mathrm{mM}$ B-NADPH, $0.1 \mathrm{mM}$ nitroblue tetrazolium and $0.3 \%$ Triton $\mathrm{X}-100$ at $37^{\circ} \mathrm{C}$ for $1 \mathrm{~h}$. The reaction was terminated by rinsing the cultures with cold PBS. After exposure of cultures to $0.3 \% \mathrm{H}_{2} \mathrm{O}_{2}$ in PBS/methanol (1 : 1) for $30 \mathrm{~min}$, subsequent procedures for $\mathrm{TH}$ immunohistochemistry, starting with $10 \%$ fetal calf serum treatment, were performed as described above.

\subsection{Statistical Analysis}

The data were analyzed by Student's t-test or one factorial ANOVA followed by a post hoc Dunnett t-test. A P value less than 0.05 was considered to be statistically significant. 


\section{Results}

\subsection{Dose-dependent death of TH-positive (dopamine) neurons}

When various concentrations $(1-100 \mu \mathrm{M})$ of PQ were applied for 2 days, dopamine neurons in midbrain cultured slices decreased in a dose-dependent manner (Figs. $1 \& 2$ ). The treatments with greater than $50 \mu \mathrm{M}$ PQ doses showed significantly reduced TH-positive cells $(P<0.01)$. However, the treatment with lower doses than $25 \mu \mathrm{M} P Q$ did not affect the dopamine neurons.

\subsection{Effects of GBR-12909, L-NAME, DNQX and cycloheximide}

A significant TH positive cell death in the slices was observed by the exposure of 50 $\mu \mathrm{M}$ PQ (Figs $1 \&$ 2). We tested the effects of $10 \mu \mathrm{M}$ GBR-12909, $200 \mu \mathrm{M}$ L-NAME, 10 $\mu \mathrm{M}$ DNQX and $10 \mu \mathrm{M}$ cycloheximide on the cell toxicity induced by the exposure of $50 \mu \mathrm{M}$ PQ. These chemicals themselves without cycloheximide did not affect the number of $\mathrm{TH}$ positive cells in the slice cultures (data not shown). Cycloheximide at $10 \mu \mathrm{M}$ slightly reduced TH positive neurons in slices (data not shown) The co-exposure with GBR-12909 or L-NAME rescued the dopamine neuronal toxicity induced by the $50 \mu \mathrm{M} P Q(P<0.01$, Fig. 3). However, the treatment with $10 \mu \mathrm{M}$ each of DNQX and cycloheximide did not rescue dopaminergic cell death (Fig. 3).

\subsection{Sequential treatment of $P Q$ and $M P P+$}

The number of $\mathrm{TH}$ positive neurons was not reduced by the treatment with $10 \mu \mathrm{M} P Q$ (Figs. 1, $4 \& 5$ ) or $1 \mu \mathrm{M} \mathrm{MPP}+$ (Figs. $4 \& 5$ ). However, the sequential treatments with 10 
$\mu \mathrm{M} P Q$ for 2 days and $1 \mu \mathrm{M} \mathrm{MPP}^{+}$for 1 day dramatically killed dopamine cells $(\mathrm{P}<0.01$, Figs. 4 \& 5). This toxicity induced by the sequential treatments with PQ and MPP ${ }^{+}$was prevented by the treatment with $20 \mu \mathrm{M}$ MK-801, $200 \mu \mathrm{M}$ L-NAME, $100 \mu \mathrm{M}$ caspase 9 inhibitor, $100 \mu \mathrm{M}$ caspase 3 inhibitor or $10 \mu \mathrm{M}$ cycloheximide $(\mathrm{P}<0.01)$, but not by $10 \mu \mathrm{M}$ DNQX (Fig. 4). These chemicals themselves without cycloheximide did not affect the number of TH positive cells in the slice cultures (data not shown).

3.4. Effects of L-deprenyl and D2/3 agonists on the neurotoxicity induced by the sequential treatments with $P Q$ and $M P P+$

The reduction of viable TH-positive dopaminergic neurons induced by the sequential treatments with $10 \mu \mathrm{M}$ PQ and $1 \mu \mathrm{M} \mathrm{MPP}^{+}$in the midbrain slice cultures was significantly rescued by the co-treatment with $10 \mu \mathrm{M}$ cabergoline, $50 \mu \mathrm{M}$ talipexole or $2 \mu \mathrm{M}$ L-deprenyl $(P<0.05$ or 0.01 , Figs. $5 \& 6)$. These drugs themselves did not show any effect on the number of TH positive cells in the slice cultures (data not shown).

\subsection{Distribution of NOS-containing neurons}

It was essential to confirm that NOS containing neurons were co-expressed or around the dopaminergic neurons. $\mathrm{NADH}$ diaphorase histochemistry is a useful marker in the central nervous system that contains NOS (Dawson et al., 1991). We processed slice cultures for double staining with NADPH diaphorase and TH in the midbrain slice cultures. NOS containing neurons showed scattered distributions throughout the midbrain slices. However, the neurons expressing NOS were also identified inside and around the regions where dopamine neurons were packed (Fig. 7). Most NADPH diaphorase positive cells displayed no TH immunoreactivity, but several double-labeled cells were also observed (Fig. 7). 


\section{Discussion}

Recently, various studies have increased interest in the possibility that environmental neurotoxins such as pesticides may be related to the development of PD (Betarbet et al., 2000; Giasson and Lee, 2000; Ritz and Yu, 2000; Thiruchelvam et al., 2000a), although the major risk factor is aging. PQ is one of the possible herbicides that are involved in PD, since a strong correlation has been found between the incidence of the disease and the amount of PQ used (Lanska, 1997; Liou et al., 1997; Rajput et al., 1987; Ritz and Yu, 2000). The concurrent exposure to PQ and some other chemicals could perturb the balance of homeostatic defense mechanisms leading to neuronal degeneration, even if exposure to each pesticide may be insufficient to induce overt effects (Thiruchelvam et al., 2000a). In addition, repeated systematic PQ injections killed dopaminergic neurons in the substantia nigra pars compacta (McCormack et al., 2002), and dopamine depletion in the striatal and midbrain (Shimizu et al., 2003). However, the mechanism of the neuronal toxicity occurring under continuous exposure to low levels of PQ for a long period has not been determined. We previously reported using the striatal microdialysis method that PQ stimulated glutamate efflux to initiate excitotoxicity, resulting in evoked depolarization of NMDA receptor channels (Shimizu et al., 2003). The influx of $\mathrm{Ca}^{2+}$ into cells stimulates NOS, followed by the released NO that would diffuse to dopaminergic terminals and induce mitochondrial dysfunction, causing continuous and long-lasting dopamine overflow (Shimizu et al., 2003). The elevation of extracellular glutamate concentration evoked by MPP+, particularly under conditions of reduced dopaminergic ATP production, is expected to result in excessive stimulation of NMDA and AMPA/kinate receptors leading to an influx of $\mathrm{Ca}^{2+}$ and excitotoxicity (Greene and Greenamyre, 1996; Novelli et al., 1988). In similar fashion, PQ could cause excitotoxicity of dopaminergic neurons. PQ is known to be an inhibitor of mitochondrial respiration chain (Tawara et al., 1996).

In this study, we confirmed the dopaminergic toxicity mechanisms in organotypic 
midbrain cultures. The exposure of PQ reduced dopaminergic neurons in midbrain cultured slices in a dose-dependent manner. However, relatively high doses of PQ required to reduce dopaminergic neurons. The significant dopaminergic neuronal death in the slices induced by $50 \mu \mathrm{M}$ PQ was prevented by the co-treatment with GBR-12909, a dopamine transporter inhibitor, indicating the transport of $\mathrm{PQ}$ into dopamine neurons could be essential to induce cell toxicity. We also showed that the neurons expressing NOS were found inside and around the regions where dopamine neurons were packed. In contrast to our previous findings (Shimizu et al., 2003) by the striatal microdialysis study, the DNQX treatment, a non-NMDA receptor antagonist, could not rescue the dopaminergic cell death. The dopaminergic neuronal toxicity was not attenuated by the treatment with cycloheximide, a protein synthesis inhibitor. This result suggested that dopaminergic cell death caused by PQ alone might not be apoptosis.

The number of dopaminergic neurons was not reduced by the treatment with $10 \mu \mathrm{M}$ PQ or $1 \mu \mathrm{M} \mathrm{MPP}^{+}$. However, the sequential treatments with $10 \mu \mathrm{M}$ PQ for 2 days and $1 \mu \mathrm{M} \mathrm{MPP}+$ for 1 day dramatically killed dopaminergic neurons. This result strongly indicates that the exposure to a low dose of PQ could lead to the vulnerability of dopaminergic neurons. The toxicity induced by the sequential treatments with PQ and $\mathrm{MPP}^{+}$was prevented by the treatment with MK-801, an NMDA receptor inhibitor, LNAME, suggesting the excitotoxic pathway involved in NOS activation would be the mechanism in this sequential toxicity, although DNQX could not attenuate the toxicity. Interestingly, it was reported that the most ventral midbrain dopamine neurons exhibit glutamate immunoreactivity (Rayport, 2001). Glutamate and reactive oxygen species (ROS) including NO have been postulated to play pivotal roles in the pathogenesis of the neuronal cell loss that is associated with several neurological disease states including PD. $\mathrm{MPP}^{+}$is also suggested to induce the glutamate-NO mediated cytotoxicity against dopaminergic neurons in the cultured rat mesencephalon (Sawada et al., 1996a; Sawada et al., 1996b). This dopaminergic cell death induced by the sequential exposure of lower doses of PQ and $\mathrm{MPP}^{+}$was completely inhibited by the co-treatment of caspase 9 inhibitor, 
caspase 3 inhibitor or cycloheximide. These results indicate that the apoptotic cell signaling would be involved in the dopaminergic cell death process induced by the sequential treatments with PQ and MPP ${ }^{+}$. In addition, PQ markedly accelerates the $\alpha$-synuclein fibril formation in a dose-dependent fashion (Manning-Bog et al., 2002; Uversky et al., 2001). Recently, $\alpha$-synuclein-containing aggregates represent a feature of a variety of neurodegenerative disorders, including PD.

The cell death induced by the sequential treatments of PQ and MPP ${ }^{+}$was rescued by dopamine D2/3 agonists and L-deprenyl. D2/3 agonists protect cells from calcium influx, nitric oxide, and peroxynitrite toxicity, which are suggested to be the mediators of glutamate toxicity (see the references cited in Le and Jankovic, 2001). These neuroprotective effects of dopamine agonists are suggested to be mainly mediated by presynaptic D3 receptors (Ling et al., 1999). Dopamine D2/3 agonists also reduce the expression of NR1 mRNA in the caudate (Canals et al., 2001). L-Deprenyl prevents the decrease in the mitochondrial membrane potential caused by withdrawal of trophic support, resulting in the prevention of apoptosis (Wadia et al., 1998), and may rescue neurons from dopaminergic neurotoxins, including $\mathrm{MPP}^{+}$, by directly influencing mitochondrial electron transport (Matsubara et al., 2001). Indeed, we previously reported that L-deprenyl prevents the toxicity of dopaminergic terminals in the striatum (Shimizu et al., 2003). L-Deprenyl also attenuates the nigrostriatal damage induced by the intranigral administration of PQ (Liou et al., 2001). Recently, deprenyl and dopamine D2 receptor agonist have been reported that they increase the levels of neurotrophins (Guo et al., 2002; Mizuta et al., 2000; Tatton et al., 2002). Together with the present findings, it is suggested that dopamine D2/3 agonists and Ldeprenyl could be effective to prevent the neuronal damage caused by constant exposure to low doses of PQ, which might be involved in the etiology of PD.

From the present results, PQ stimulated midbrain neurons to release glutamate, followed by the activation of NMDA receptor-channels. An influx of $\mathrm{Ca}^{2+}$ would stimulate NOS activities in NOS expressed neurons that existed around and inside dopamine neurons. NO can diffuse to the dopaminergic neurons and then interact with ROS from 
other sources to generate highly reactive peroxynitrite (Beckman and Koppenol, 1996), which uncouples mitochondrial electron transport enhancing mitochondrial production of free radicals (Dykens, 1994). This PQ toxicity led to the vulnerability of dopaminergic neurons. Indeed, significant cell death was induced by the sequential treatments with lower doses of PQ and $\mathrm{MPP}^{+}$. The present results strongly support that a constant exposure to low levels of PQ could lead to the vulnerability of dopaminergic neurons in the nigrostriatal system, and might potentiate neurodegeneration caused by the exposure of other substances, such as endogenous dopamine toxins, and aging. This also suggests that PQ could be considered an exogenous neurotoxin involved in the etiology of PD.

\section{Acknowledgments}

The authors wish to express their cordial thanks to Dr. Akaike, Dr. Katsuki and Mr. Shibata (Kyoto University, Japan) for technical suggestions for the slice culture. This work was supported in part by grants from the Ministry of Education, Science, Sports, Culture and Technology, Japan and from the Uehara Foundation. 


\section{References}

Beal, M.F., 1998. Excitotoxicity and nitric oxide in Parkinson's disease pathogenesis. Ann Neurol. 44, S110 - 114.

Beckman, J.S., Koppenol, W.H., 1996. Nitric oxide, superoxide, and peroxynitrite: the good, the bad, and ugly. Am. J. Physiol. 271, C1424 - 1437.

Betarbet, R., Sherer, T.B., MacKenzie, G., Garcia-Osuna, M., Panov, A.V., Greenamyre, J.T., 2000. Chronic systemic pesticide exposure reproduces features of Parkinson's disease. Nat. Neurosci. 3, 1301 - 1306.

Brooks, A.I., Chadwick, C.A., Gelbard, H.A., Cory-Slechta, D.A., Federoff, H.J., 1999. Paraquat elicited neurobehavioral syndrome caused by dopaminergic neuron loss. Brain Res.. 823, 1 - 10.

Canals, S., Casarejos, M.J., de Bernardo, S., Rodriguez-Martin, E., Mena, M.A., 2001. Glutathione depletion switches nitric oxide neurotrophic effects to cell death in midbrain cultures: implications for Parkinson's disease. J. Neurochem. 79, 1183 1195.

Corasaniti, M.T., Bagetta, G., Rodino, P., Gratteri, S., Nistico, G., 1992. Neurotoxic effects induced by intracerebral and systemic injection of paraquat in rats. Hum. Exp. Toxicol. 11, 535 - 539.

Corasaniti, M.T., Strongoli, M.C., Rotiroti, D., Bagetta, G., Nistico, G., 1998. Paraquat: a useful tool for the in vivo study of mechanisms of neuronal cell death. Pharmacol. Toxicol. 83, 1 - 7 .

Corrigan, F.M., Wienburg, C.L., Shore, R.F., Daniel, S.E., Mann, D., 2000. Organochlorine insecticides in substantia nigra in Parkinson's disease. $\mathbf{J}$ Toxicol. Environ. Health A 59, 229 - 234.

Dawson, T.M., Bredt, D.S., Fotuhi, M., Hwang, P.M., Snyder, S.H., 1991. Nitric oxide synthase and neuronal NADPH diaphorase are identical in brain and peripheral tissues. Proc. Natl. Acad. Sci. USA 88, 7797 - 7801. 
Dawson, V.L., Dawson, T.M., 1998. Nitric oxide in neurodegeneration. Prog Brain Res $118,215-229$.

Dugan, L., Choi, D.W., 1999. Hypoxic-ischemic brain injury and oxidative stress. In: Siegel, G.J., Agranoff, B.W., Albers, R.W., Fisher, S.K., Uhler, M.D. (Eds.), Basic Neurochemistry, Lipincott-Raven, Philadelphia, pp. 712 - 729.

Dykens, J.A., 1994. Isolated cerebral and cerebellar mitochondria produce free radicals when exposed to elevated $\mathrm{Ca}^{2+}$ and $\mathrm{Na}^{+}$: implications for neurodegeneration. J. Neurochem. 63, 584 - 591.

Fleming, L., Mann, J.B., Bean, J., Briggle, T., Sanchez-Ramos, J.R., 1994. Parkinson's disease and brain levels of organochlorine pesticides. Ann. Neurol. 36, 100 - 103.

Gatto, E.M., Riobo, N.A., Carreras, M.C., Chernavsky, A., Rubio, A., Satz, M.L., Poderoso, J.J., 2000. Overexpression of neutrophil neuronal nitric oxide synthase in Parkinson's disease. Nitric Oxide 4, 534 - 539.

Giasson, B.I., Lee, V.M., 2000. A new link between pesticides and Parkinson's disease. Nat. Neurosci. 3, 1227 - 1228.

Greene, J.G., Greenamyre, J.T., 1996. Bioenergetics and glutamate excitotoxicity. Prog. Neurobiol. 48, 613 - 634.

Guo, H., Tang, Z., Yu, Y., Xu, L., Jin, G., Zhou, J., 2002. Apomorphine induces trophic factors that support fetal rat mesencephalic dopaminergic neurons in cultures. Eur. J. Neurosci. 16, 1861-1870.

Katsuki, H., Tomita, M., Takenaka, C., Shirakawa, H., Shimazu, S., Ibi, M., Kume, T., Kaneko, S., Akaike, A., 2001. Superoxide dismutase activity in organotypic midbrain-striatum co-cultures is associated with resistance of dopaminergic neurons to excitotoxicity. J. Neurochem. 76, $1336-1345$.

Kotake, Y., Tasaki, Y., Makino, Y., Ohta, S., Hirobe, M., 1995. 1-Benzyl-1,2,3,4tetrahydroisoquinoline as a parkinsonism-inducing agent: a novel endogenous amine in mouse brain and parkinsonian CSF. J. Neurochem. 65, 2633 - 2638.

Langston, J.W., 1998. Epidemiology versus genetics in Parkinson's disease: progress in 
resolving an age-old debate. Ann .Neurol. 44, S45 - 52.

Lanska, D.J., 1997. The geographic distribution of Parkinson's disease mortality in the United States. J. Neurol. Sci. 150, 63 - 70.

LaVoie, M.J., Hastings, T.G., 1999. Peroxynitrite- and nitrite-induced oxidation of dopamine: implications for nitric oxide in dopaminergic cell loss. J. Neurochem. 73, $2546-2554$

Le, W.D., Jankovic, J., 2001. Are dopamine receptor agonists neuroprotective in Parkinson's disease? Drugs Aging 18, 389 - 396.

Ling, Z.D., Robie, H.C., Tong, C.W., Carvey, P.M., 1999. Both the antioxidant and D3 agonist actions of pramipexole mediate its neuroprotective actions in mesencephalic cultures. J. Pharmacol. Exp. Ther. 289, 202 - 210.

Liou, H.H., Chen, R.C., Chen, T.H., Tsai, Y.F., Tsai, M.C., 2001. Attenuation of paraquat-induced dopaminergic toxicity on the substantia nigra by (-)-deprenyl in vivo. Toxicol. Appl. Pharmacol. 172, 37 - 43.

Liou, H.H., Tsai, M.C., Chen, C.J., Jeng, J.S., Chang, Y.C., Chen, S.Y., Chen, R.C., 1997. Environmental risk factors and Parkinson's disease: a case-control study in Taiwan. Neurology 48, $1583-1588$.

Maeda, T., Ibi, M., Shimazu, S., Akaike, A., 1998. Co-culture with the striatum attenuates $\mathrm{N}$-methyl-D-aspartate cytotoxicity in dopaminergic neurons of rat mesencephalic slice cultures. Jpn. J. Pharmacol. 77, 161 - 167.

Manning-Bog, A.B., McCormack, A.L., Li, J., Uversky, V.N., Fink, A.L., Di Monte, D.A., 2002. The herbicide paraquat causes up-regulation and aggregation of alphasynuclein in mice: paraquat and alpha-synuclein. J. Biol. Chem. 277, 1641 - 1644.

Maruyama, Y., Abe, T., Tohgi, H., Dostert, P., Naoi, M., 1996. A dopaminergic neurotoxin, (R)-N-methylsalsolinol, increases in parkinsonian cerebrospinal fluid. Ann. Neurol. 40, 119 - 122.

Matsubara, K., Kobayashi, S., Kobayashi, Y., Yamashita, K., Koide, H., Hatta, M., Iwamoto, K., Tanaka, O., Kimura, K., 1995. ß-Carbolinium cations, endogenous 
$\mathrm{MPP}^{+}$analogs, in the lumbar cerebrospinal fluid of parkinsonian patients. Neurology $45,2240-2245$.

Matsubara, K., Senda, T., Uezono, T., Awaya, T., Ogawa, S., Chiba, K., Shimizu, K., Hayase, N., Kimura, K., 2001. L-Deprenyl prevents the cell hypoxia induced by dopaminergic neurotoxins, $\mathrm{MPP}^{+}$and beta-carbolinium: a microdialysis study in rats. Neurosci. Lett. 302, 65 - 68.

McCormack, A.L., Thiruchelvam, M., Manning-Bog, A.B., Thiffault, C., Langston, J.W., Cory-Slechta, D.A., Di Monte, D.A., 2002. Environmental risk factors and Parkinson's disease: selective degeneration of nigral dopaminergic neurons caused by the herbicide paraquat. Neurobiol. Dis. 10, 119 - 127.

Mizuta, I., Ohta, M., Ohta, K., Nishimura, M., Mizuta, E., Hayashi, K., Kuno, S., 2000. Selegiline and desmethylselegiline stimulate NGF, BDNF, and GDNF synthesis in cultured mouse astrocytes. Biochem. Biophys. Res. Commun. 279, 751-755.

Moghal, S., Rajput, A.H., D'Arcy, C., Rajput, R., 1994. Prevalence of movement disorders in elderly community residents. Neuroepidemiology 13, 175 - 178.

Nakamura, K., Bindokas, V.P., Kowlessur, D., Elas, M., Milstien, S., Marks, J.D., Halpern, H.J., Kang, U.J., 2001. Tetrahydrobiopterin scavenges superoxide in dopaminergic neurons. J. Biol. Chem. 276, 34402 - 34407.

Novelli, A., Reilly, J.A., Lysko, P.G., Henneberry, R.C., 1988. Glutamate becomes neurotoxic via the N-methyl-D-aspartate receptor when intracellular energy levels are reduced. Brain Res. 451, 205 - 212.

Obata, T., Yamanaka, Y., 2001. Nitric oxide enhances $\mathrm{MPP}^{+}$-induced hydroxyl radical generation via depolarization activated nitric oxide synthase in rat striatum. Brain Res. 902, 223 - 228.

Olanow, C.W., Tatton, W.G., 1999. Etiology and pathogenesis of Parkinson's disease. Annu. Rev. Neurosci. 22, 123 - 144.

Rajput, A.H., Uitti, R.J., Stern, W., Laverty, W., O'Donnell, K., O'Donnell, D., Yuen, W.K., Dua, A., 1987. Geography, drinking water chemistry, pesticides and herbicides and 
the etiology of Parkinson's disease. Can. J. Neurol. Sci. 14, 414 - 418.

Rayport, S., 2001. Glutamate is a cotransmitter in ventral midbrain dopamine neurons. Parkinsonism Relat. Disord. 7, 261 - 264.

Ritz, B., Yu, F., 2000. Parkinson's disease mortality and pesticide exposure in California 1984-1994. Int. J. Epidemiol. 29, 323 - 329.

Sanchez-Ramos, J., Facca, A., Basit, A., Song, S., 1998. Toxicity of dieldrin for dopaminergic neurons in mesencephalic cultures. Exp. Neurol. 150, 263 - 271.

Sawada, H., Shimohama, S., Tamura, Y., Kawamura, T., Akaike, A., Kimura, J., 1996a. Methylphenylpyridium ion $\left(\mathrm{MPP}^{+}\right)$enhances glutamate-induced cytotoxicity against dopaminergic neurons in cultured rat mesencephalon. J. Neurosci. Res. 43, 55 - 62.

Sawada, H., Shimohama, S., Kawamura, T., Akaike, A., Kitamura, Y., Taniguchi, T., Kimura, J., 1996b. Mechanism of resistance to NO-induced neurotoxicity in cultured rat dopaminergic neurons. J. Neurosci. Res. 46, 509 - 518.

Seidler, A., Hellenbrand, W., Robra, B.P., Vieregge, P., Nischan, P., Joerg, J., Oertel, W.H., Ulm, G., Schneider, E., 1996. Possible environmental, occupational, and other etiologic factors for Parkinson's disease: a case-control study in Germany. Neurology 46, 127 5- 1284.

Semchuk, K.M., Love, E.J., Lee, R.G., 1993. Parkinson's disease: a test of the multifactorial etiologic hypothesis. Neurology 43, $1173-1180$.

Shimizu, K., Matsubara, K., Ohtaki, K., Fujimaru, S., Saito, O., Shiono, H., 2003. Paraquat induces long-lasting dopamine overflow through excitotoxic pathway in the striatum of freely moving rats. Brain Res. (accepted).

Shimizu, K., Ohtaki, K., Matsubara, K., Aoyama, K., Uezono, T., Saito, O., Suno, M., Ogawa, K., Hayase, N., Kimura, K., Shiono, H., 2001. Carrier-mediated processes in blood-brain barrier penetration and neural uptake of paraquat. Brain Res. 906, $135-142$

Simonian, N.A., Coyle, J.T., 1996. Oxidative stress in neurodegenerative diseases. Annu. Rev. Pharmacol. Toxicol. 36, 83 - 106. 
Stoppini, L., Buchs, P.A., Muller, D., 1991. A simple method for organotypic cultures of nervous tissue. J. Neurosci. Methods 37, 173 - 182.

Tanner, C.M., Ottman, R., Goldman, S.M., Ellenberg, J., Chan, P., Mayeux, R., Langston, J.W., 1999. Parkinson disease in twins: an etiologic study. Jama 281, 341 - 346.

Tatton, W.G., Chalmers-Redman, R.M., Ju, W.J., Mammen, M., Carlile, G.W., Pong, A.W., Tatton, N.A., 2002. Propargylamines induce antiapoptotic new protein synthesis in serum- and nerve growth factor (NGF)-withdrawn, NGF-differentiated PC-12 cells. J. Pharmacol. Exp. Ther. 301, 753-764.

Tawara, T., Fukushima, T., Hojo, N., Isobe, A., Shiwaku, K., Setogawa, T., Yamane, Y., 1996. Effects of paraquat on mitochondrial electron transport system and catecholamine contents in rat brain. Arch. Toxicol. 70, 585 - 589.

Thiruchelvam, M., Richfield, E.K., Baggs, R.B., Tank, A.W., Cory-Slechta, D.A., 2000a. The nigrostriatal dopaminergic system as a preferential target of repeated exposures to combined paraquat and maneb: implications for Parkinson's disease. J. Neurosci. 20, $9207-9214$.

Thiruchelvam, M., Brockel, B.J., Richfield, E.K., Baggs, R.B., Cory-Slechta, D.A., 2000b. Potentiated and preferential effects of combined paraquat and maneb on nigrostriatal dopamine systems: environmental risk factors for Parkinson's disease? Brain Res. $873,225-234$.

Uversky, V.N., Li, J., Fink, A.L., 2001. Pesticides directly accelerate the rate of alphasynuclein fibril formation: a possible factor in Parkinson's disease. FEBS Lett. 500, $105-108$.

Wadia, J.S., Chalmers-Redman, R.M.E., Ju, W.J.H., Carlile, G.W., Phillips, J.L., Fraser, A.D., Tatton, W.G., 1998. Mitochondrial membrane potential and nuclear changes in apoptosis caused by serum and nerve growth factor withdrawal: time course and modification by (-)-deprenyl. J. Neurosci. 18, 932 - 947. 
Legends for figures

Fig. 1. Dose-dependent effects of PQ on TH positive neurons in midbrain slices. TH positive neurons in control slices are expressed as $100 \%$. PQ was exposed to the slices for 2 days. Note that the treatment with a $10 \mu \mathrm{M}$ of PQ exposure did not affected the THpositive neurons. Values are presented as means $\AA$ \} SEM of 10 slice cultures. $\quad * P<$ 0.01 .

Fig. 2. Typical photographs showing the dose-dependent toxicity of PQ exposure on TH positive neurons in midbrain slices. PQ was exposed to the slices for 2 days. A: Control, B: $25 \mu \mathrm{M}, \mathrm{C}: 50 \mu \mathrm{M}$ and D: $100 \mu \mathrm{M}$.

Fig. 3. Effects of GBR-12909 $(10 \mu \mathrm{M})$, L-NAME $(200 \mu \mathrm{M})$, DNQX $(10 \mu \mathrm{M})$ and cycloheximide $(10 \mu \mathrm{M})$ on the PQ $(50 \mu \mathrm{M})$ induced dopaminergic neurotoxicity. Slices from the contralateral hemisphere from the same animal were used as controls. Control slices were also administered these drugs without the toxin treatment. TH positive neurons in control slices are expressed as $100 \%$. Data are presented as means \pm SEM of 10 slice cultures. $* \mathrm{P}<0.01$ vs control. $\dagger \mathrm{P}<0.01$ vs the neurotoxicity induced by the $50 \mu \mathrm{M}$ PQ.

Fig. 4. Effects of various antagonists on the dopaminergic toxicity induced by the sequential exposures of $\mathrm{PQ}$ and $\mathrm{MPP}^{+}$. Mid-brain slices were incubated with $10 \mu \mathrm{M} \mathrm{PQ}$ for 2 days followed by $1 \mu \mathrm{M} \mathrm{MPP}+$ for 1 day. During exposure to toxins, MK-801 (20 $\mu \mathrm{M})$, L-NAME $(10 \mu \mathrm{M})$, DNQX $(10 \mu \mathrm{M})$, caspase $9(100 \mu \mathrm{M})$, caspase $3(100 \mu \mathrm{M})$ or cycloheximide $(10 \mu \mathrm{M})$ was co-incubated. Slices from the contralateral hemisphere from the same animal were used as controls. Control slices were also administered these drugs without the toxin treatment. TH positive neurons in control slices are expressed as $100 \%$. Values are presented as means \pm SEM of 10 slice cultures. $\quad * P<0.01$ vs control. $\dagger P<$ 
0.01 vs the neurotoxicity induced by $\mathrm{PQ}$ with $\mathrm{MPP}^{+}$.

Fig. 5. Histochemical dopamine neurons rescued the dopaminergic toxicity induced by PQ with $\mathrm{MPP}^{+}$by the dopamine D2/3 agonist, cabergoline. Note that $10 \mu \mathrm{M}$ PQ or $1 \mu \mathrm{M}$ $\mathrm{MPP}^{+}$did not affect the number of TH positive neurons, but the sequential exposure of PQ and $\mathrm{MPP}^{+}$strongly killed $\mathrm{TH}$ positive neurons.

Fig. 6. Dopamine D2/3 agonists, cabergoline and talipexole, and L-deprenyl rescued the dopaminergic toxicity induced by PQ with $\mathrm{MPP}^{+}$. Slices from the contralateral hemisphere from the same animal were used as controls. Control slices were also administered these drugs without the toxin treatment. TH positive neurons in control slices are expressed as $100 \%$. Data are represented as means \pm SEM of 10 slice cultures. $* P<0.01$ vs control. $\dagger \mathrm{P}<0.05, \dagger \uparrow \mathrm{P}<0.01$ vs the neurotoxicity induced by $\mathrm{PQ}$ with $\mathrm{MPP}^{+}$.

Fig. 7. Histochemical localization of dopaminergic neurons and NOS-containing neurons in midbrain cultured slice. Dopaminergic neurons were visualized by $\mathrm{TH}-$ immunohistochemistry (brown), and NOS-containing neurons were by NADPH-diaphorase staining (blue). Several neurons were double stained for TH and NADPH diaphorase (dark bule-brown). 
Fig. 1

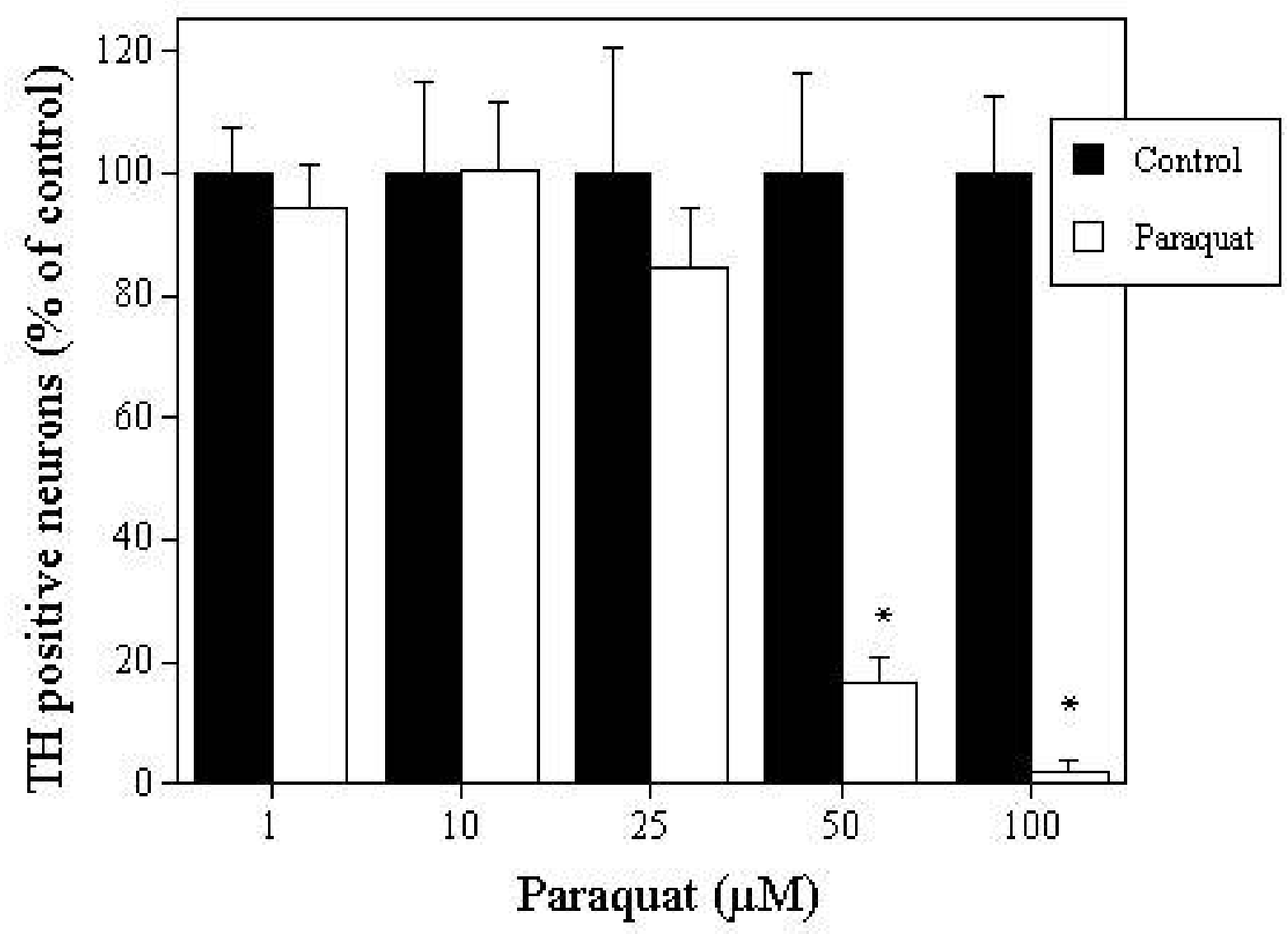


Fig. 2
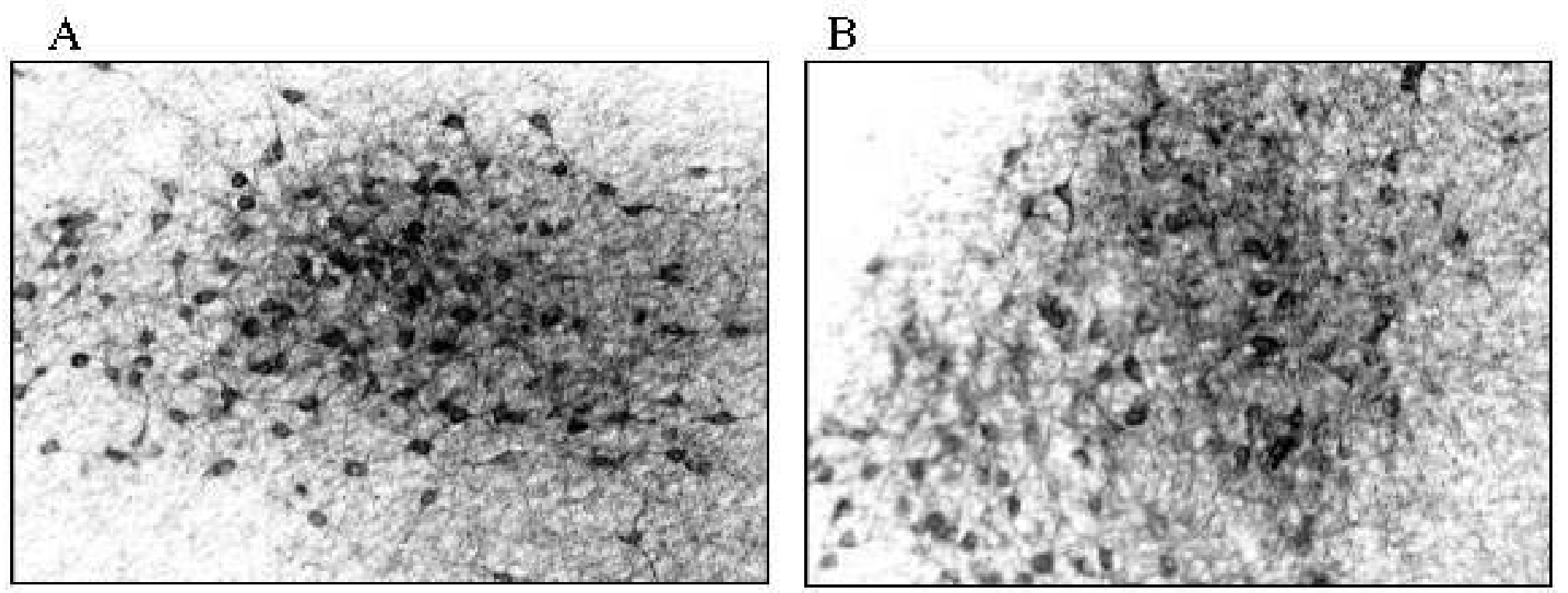

C.

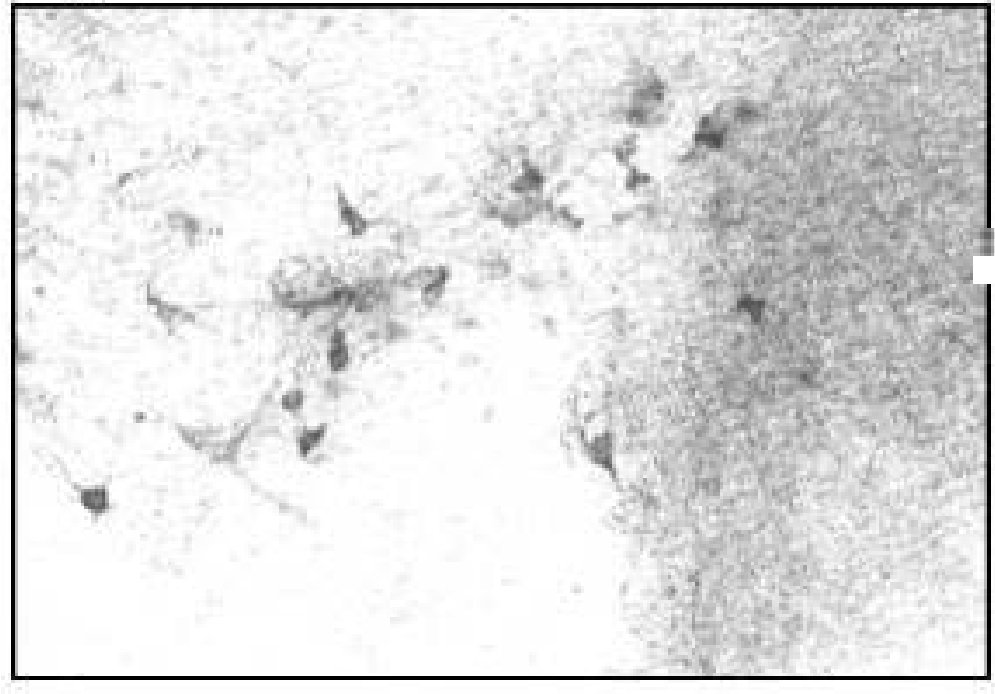

D

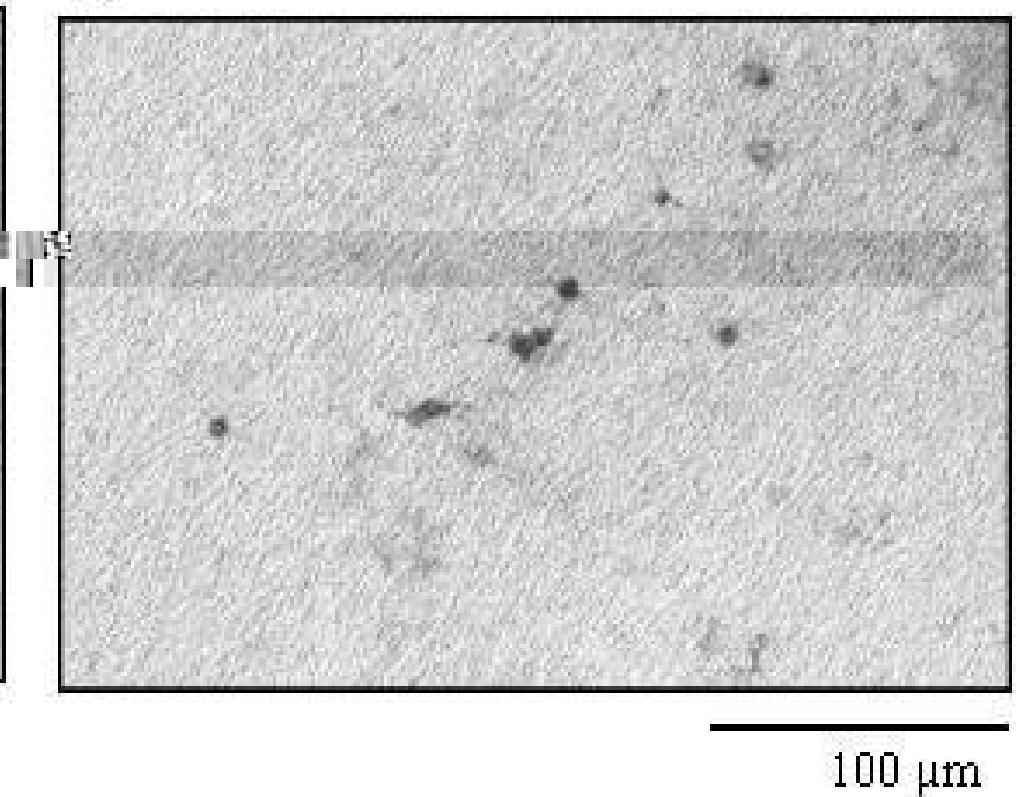


Fig. 3

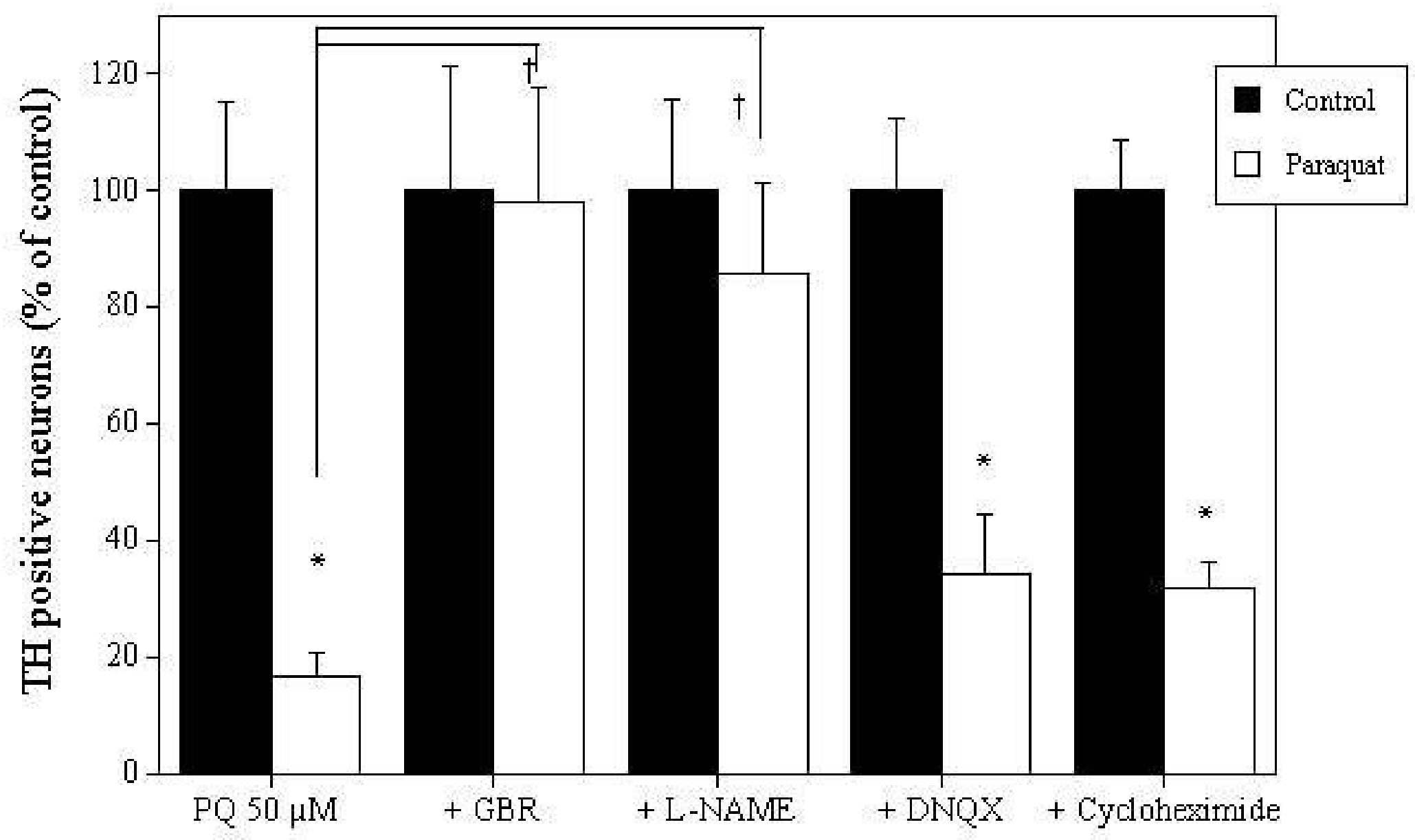


Fig. 4

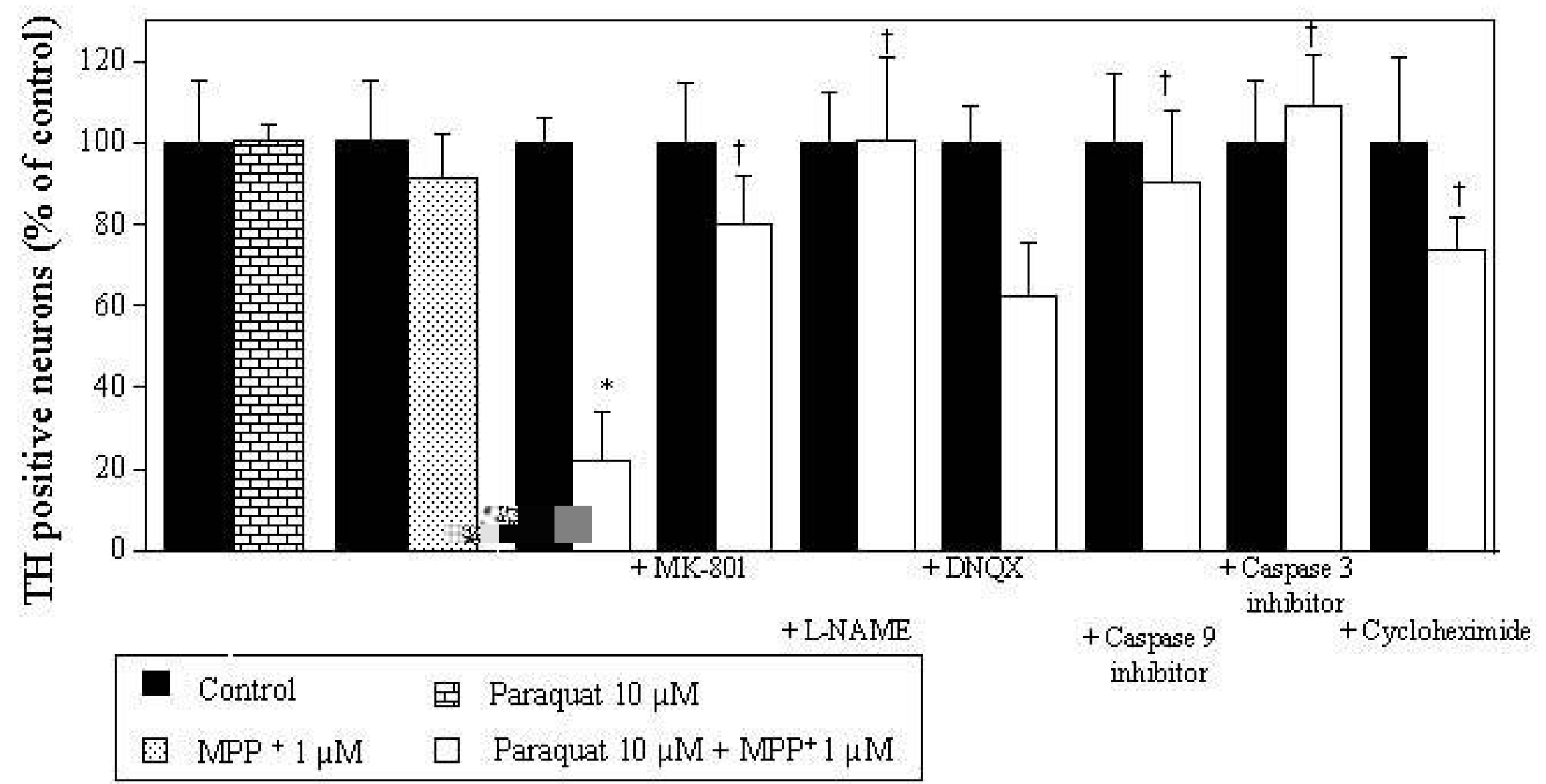


Fig. 5

A

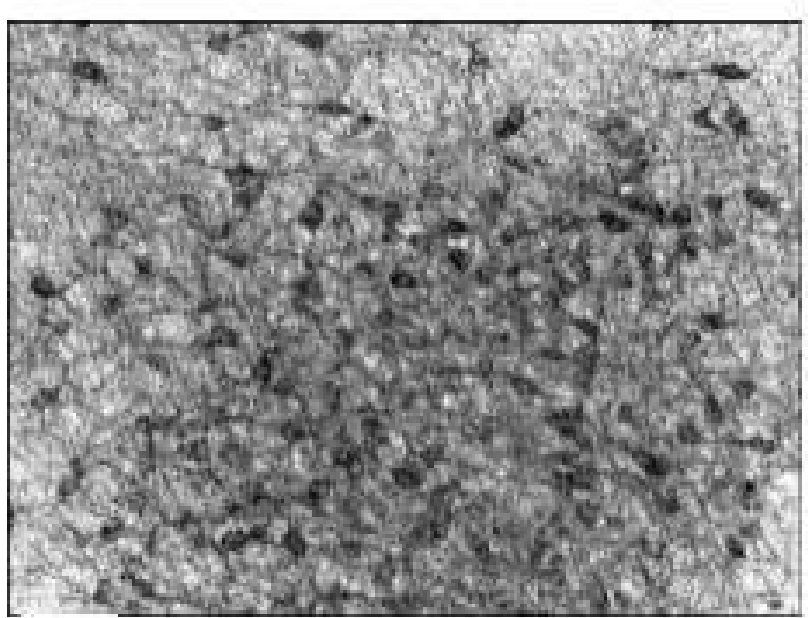

D

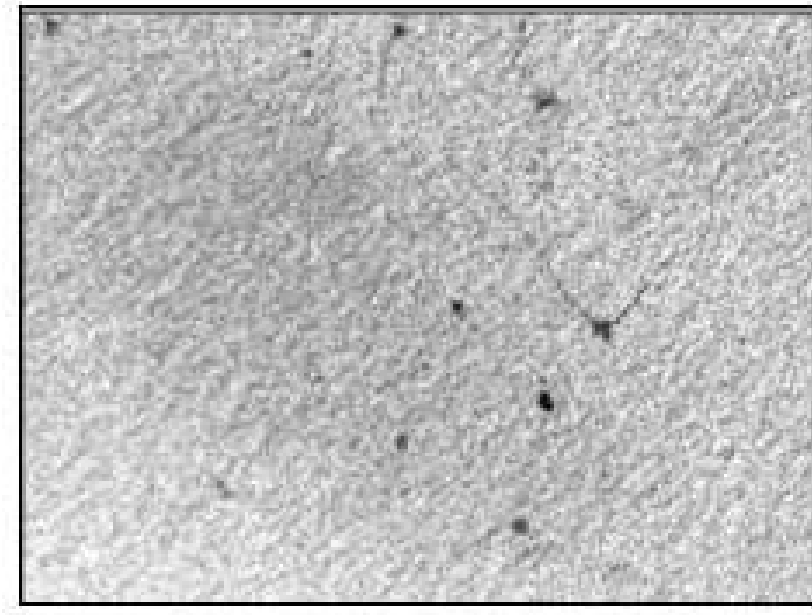

B

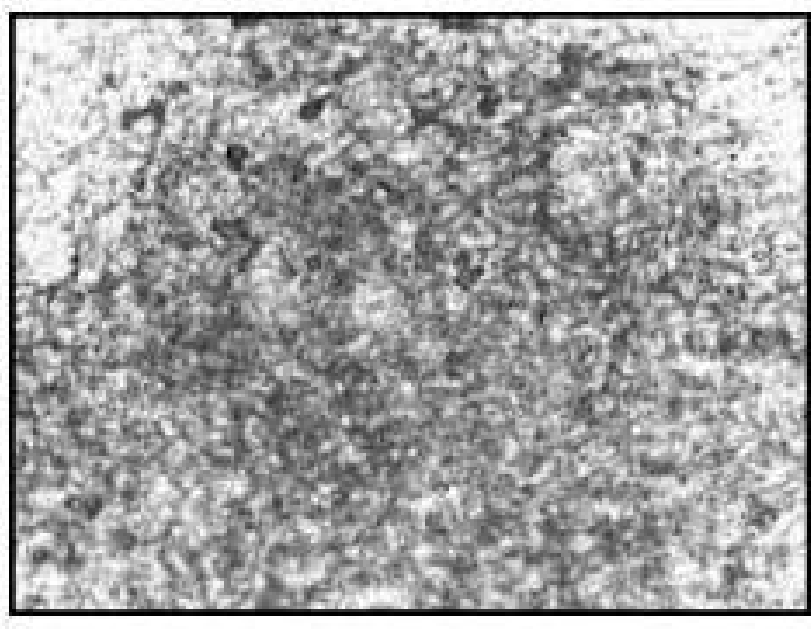

E

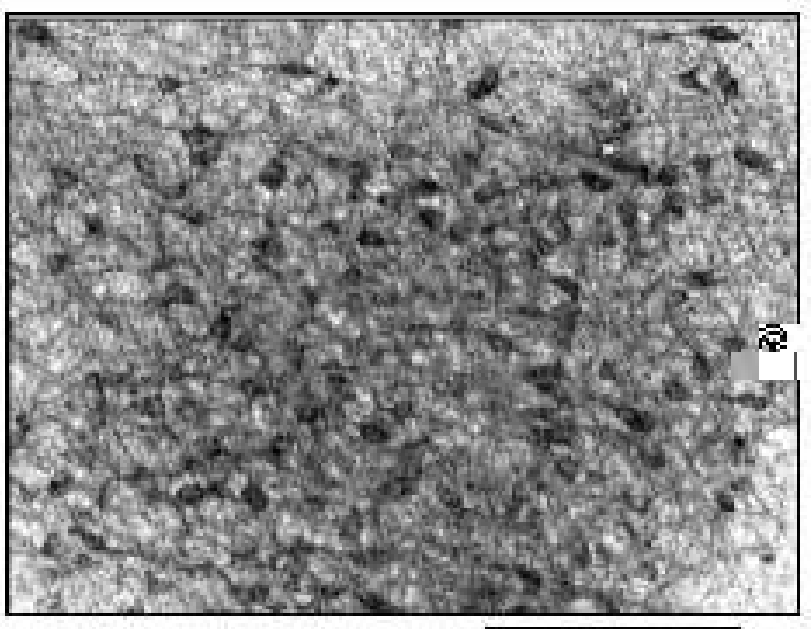

$100 \mu \mathrm{m}$
C

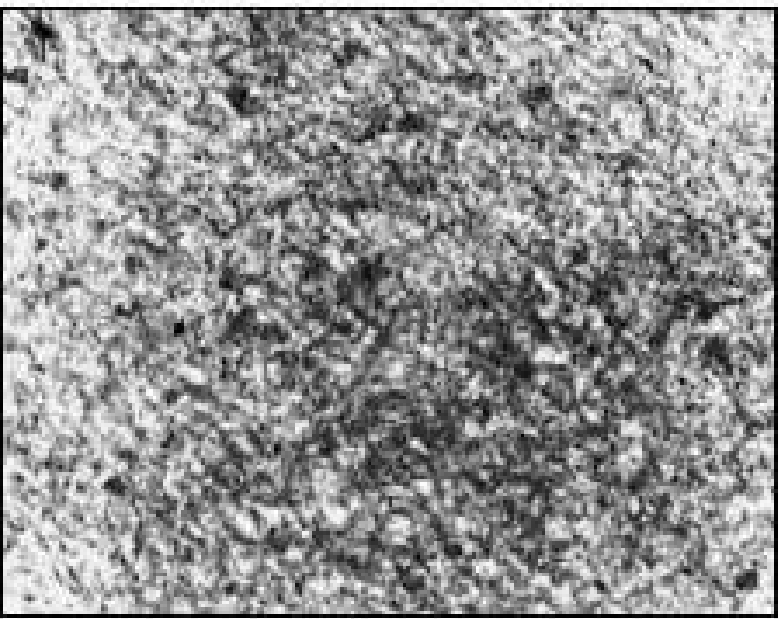

\begin{tabular}{|c|c|c|c|c|c|}
\hline Slice & A & B & C & D & $E$ \\
\hline $\begin{array}{c}\text { Paraquat } \\
(10 \mu \mathrm{M})\end{array}$ & - & + & - & + & + \\
\hline $\begin{array}{c}\mathrm{MPP}_{(1 \mu \mathrm{M})}^{+} \\
\text {(1) }\end{array}$ & - & - & + & + & + \\
\hline${ }_{\left(1 \mathrm{u} \mathrm{M}^{-}\right.}$ & & - & - & - & $-\quad+$ \\
\hline
\end{tabular}


Fig. 6

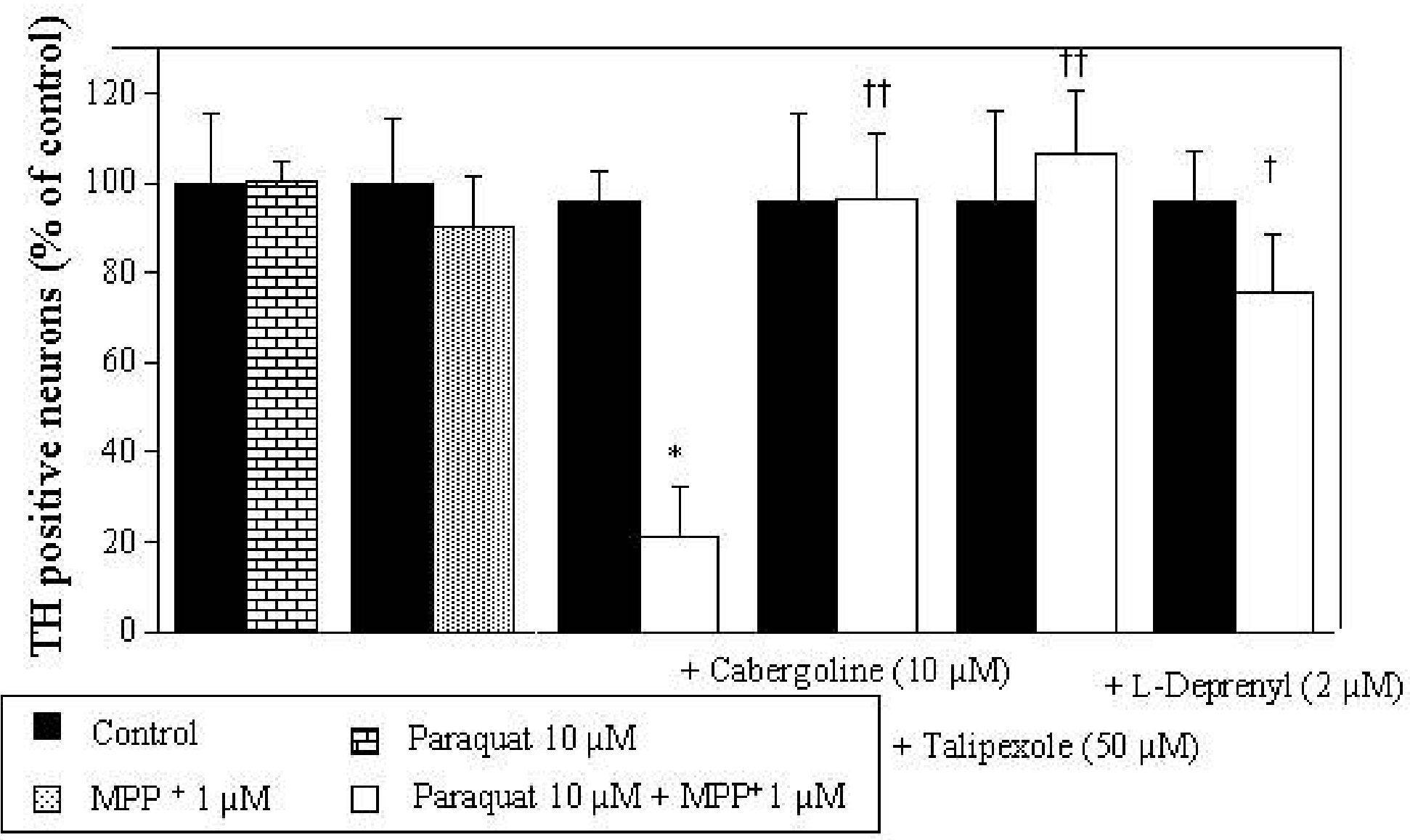


Fig. 7

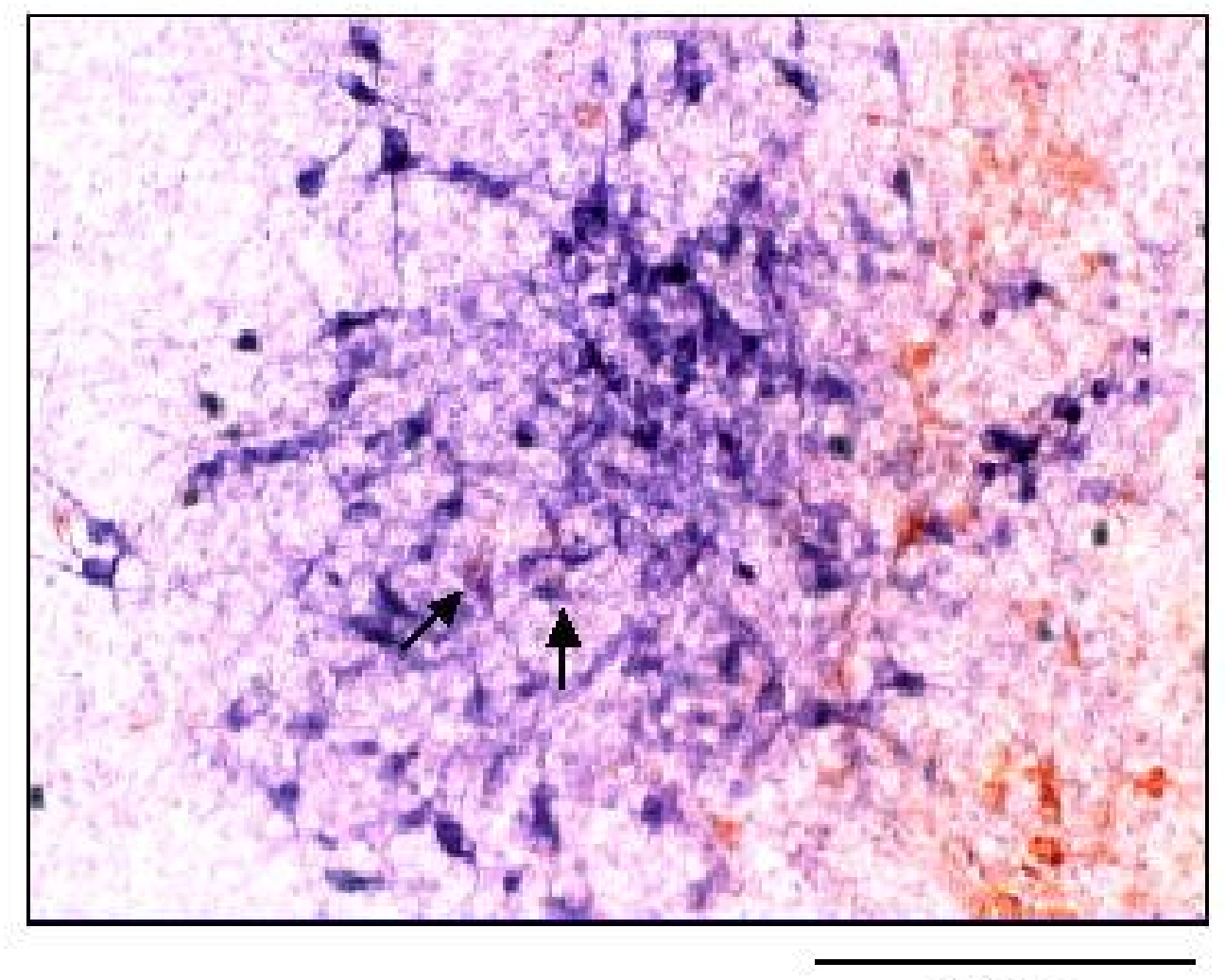

$100 \mu \mathrm{m}$ 
Fig. 8

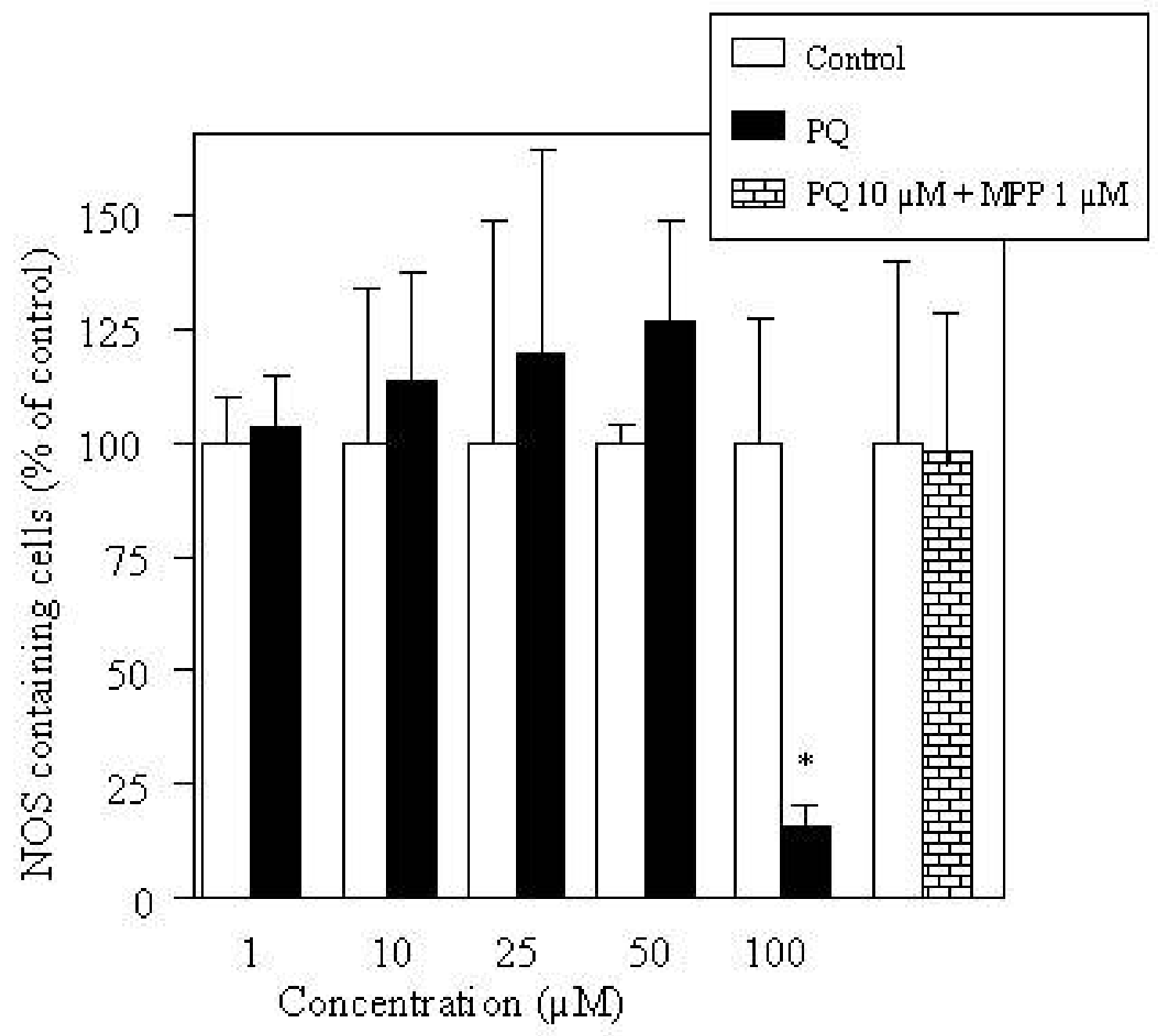

